\title{
La Révolution introuvable? ${ }^{1}$
}

Alain Renaud

Quelques difficultés très spécifiques s'attachent à la tentative de suggérer dans quelle mesure l'interprétation aronienne de Mai 68 peut rester une référence féconde. Bien que cette interprétation se soit largement poursuivie dans divers textes postérieurs à La Révolution introuvable, et ce jusqu'aux Mémoires ${ }^{2}$, on n'entend pas moins à considérer son sort pour réglé dès l'été 1968 , avec des pages qui, écrites ou dictées "à chaud », furent lues ou parcourues le plus souvent dans la même précipitation. Or $L a$ Révolution introuvable constitue à sa manière une sorte de livre maudit. Reçu dans des conditions profondément prédéterminées depuis L'Opium des intellectuels, l'essai fut plus fréquemment vitupéré que véritablement lu et, aux yeux de beaucoup, semble avoir définitivement condamné son auteur pour (une fois n'est pas coutume) flagrant délit de manque de lucidité et d'incapacité à saisir la portée réelle d'un moment d'histoire : comme le lui écrivait alors avec amabilité A. Fabre-Luce, Aron aurait ainsi rejoint clairement " le camp des mandarins et des conservateurs $»^{3}$. Il se trouve pourtant que, dans le bilan de la crise de Mai dressé, quinze ans après, par les Mémoires, l'ordre rétabli après les événements sera présenté comme différant " en mieux de l'ordre ancien»" ${ }^{4}$ de Mai, s'il en fut le critique sévère et se reconnut comme tel $^{5}$, Aron dresse donc un bilan globalement positif, quand bien même, à tort ou à raison, il en enregistre, notamment pour ce qui concerne le destin de l'Université, certains effets à ses yeux irréparables. Signe sans doute que l'interprétation esquissée "à chaud » (et non démentie, dans ses grandes lignes, par les écrits ultérieurs) n'était pas, malgré sa sévérité, malgré ses injustices éventuelles, si intégralement négative qu'à lire alors le livre (ou à ne pas le lire), on a bien voulu le croire et le faire croire. Aussi me semble-t-il qu'évaluer aujourd'hui avec pondération la lecture aronienne de Mai, c'est au fond essayer de clarifier en quel sens une condamnation certaine des pratiques de Mai et un jugement souvent accablant (parfois, sans doute, jusqu'à l'excès) à l'égard des acteurs ont pu ici coexister avec la reconnaissance d'une positivité de Mai 68 comme moment d'un processus de transformation de 1' «ordre ancien».

1 Este texto hace parte del número monográfico de la revista Pouvoirs dedicado a Mayo del 68. París, noviembre de 1986, pp. 81-89. El editor agradece tanto a Anne Sastourné editora de Pouvoirs y al autor, profesor Alain Renaud, la autorización para reproducir el artículo y ponerlo a consideración de un público académico colombiano.

2 Cf. Mémoires, Julliard, 1983, p. 470-497. On consultera aussi Les désillusions du progrès, essai sur la dialectique de la modernité, Calmann-Lévy, 1969, Préface, p. XIX-XXIII ; Plaidoyer pour l'Europe décadente, R. Laffont, 1977, Troisième Partie, IX: «Mai 68, les universités, les étudiants» (Poche/Pluriel, 1978, p. 469-484).

3 Mémoires, p. 494.

4 Ibid., p. 496.

5 La Révolution introuvable, réflexions sur les événements de Mai, Fayard, 1968, p. 134 : « Je réclame le droit de n'être pas moins sévère à l'égard des événements de Mai 1968 que le socialiste Proudhon, le libéral Tocqueville et Karl Marx l'ont été à l'égard des imitateurs de la grande Révolution, les comédiens de 1848 ». 
Dans La Révolution introuvable, Aron interprète la crise de Mai comme un "psychodrame», et jusque dans les Mémoires on retrouve l'idée qu'il s'est agi au fond d'un « carnaval» ${ }^{6}$ où, disait-il à l'été 1968, «nous avons tous (...) joué un rôle» ${ }^{7}$. Je ne reviendrai pas sur ce thème bien connu, qui évidemment ne compta pas pour rien dans l'exaspération suscitée par le livre. On verra dans un instant ce qu'il pouvait véritablement signifier. Je voudrais seulement noter qu'Aron lui-même ne s'excepte pas de la distribution des rôles ou de la répartition des masques : «Je commence par moi-même, écrit-il dans La Révolution introuvable, je vous l'ai dit, moi j'ai joué le rôle de Tocqueville, ce qui ne va pas sans quelque ridicule, mais d'autres ont joué Saint-Just, Robespierre ou Lénine, ce qui, tous comptes faits, était encore plus ridicule $»^{8}$. Je ne discuterai pas, bien sûr, ce jugement sur les différents « rôles », ni je ne m'interrogerai sur le talent plus ou moins grand avec lequel ils furent tenus (il peut y avoir d'excellents acteurs dans une mauvaise pièce, ou inversement !).

Je ne retiendrai que l'indication selon laquelle Aron a choisi, dès les événements eux-mêmes, de les lire à travers les yeux de Tocqueville, c'està-dire d'appliquer à la « révolution de Mai » les mêmes principes delecture qui avaientétéceux de Tocqueville aussi bien à l'égard des événements de février 1848 que vis-à-vis de la grande Révolution française : montrer que l'impression de rupture ou de "brèche " introduite dans l'histoire était une illusion et qu'en réalité la séquence accomplissait une logique échappant aux acteurs. C'est en effet à partir de cette décision de jouer, dans le psychodrame de Mai, le rôle de Tocqueville que se laissent dégager le plus nettement, à mon sens, les principales caractéristiques de l'interprétation aronienne. Là se joue aussi, peut-être, ce qui en elle reste fécond, au-delà même du conjoncturel et de ses humeurs. Voir les événements du point de vue Tocqueville, cela signifie en effet, au minimum, l'adoption méthodique d'un certain nombre de présupposés, dont le premier engage donc l'aveuglement des acteurs : «Je jouais le Tocqueville du 25 février 1848 qui accusait d'aveuglement son ami Ampère : vous ne comprenez rien, vous trouvez grandiose le mouvement du peuple de Paris (traduisez : vous jugez grandiose le mouvement des étudiants), mais moi je vous dis que de ce mouvement ne peut sortir que le malheur, c'est-à-dire ou un régime de droite renforcé, ou un gouvernement de style front populaire dominé par le communisme. Ainsi pensé-je depuis le premier jusqu'au dernier jour de la crise, et l'événement aboutit, selon la logique, à un gaullisme à la fois renforcé par sa majorité et affaibli par la résistance d'une partie $\mathrm{du}$ pays $»^{9}$. La reprise du schème tocquevillien (au sens où Tocqueville évoquait déjà, décrivant la France d'avant 1789, les " petits personnages » qui, « ministres étourdis », « courtisans cupides» ou roi aux "vertus inutiles et dangereuses ", «facilitent, souvent précipitent », à leur insu et parfois contre leur gré, des " événements immenses» où s'accomplit la nécessité

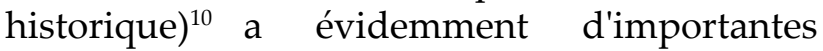
conséquences, dont la moindre n'est pas, comme d'ailleurs chez Tocqueville, une grande sévérité de ton à l'égard d'acteurs faisant l'histoire, diraiton en un autre langage, sans savoir l'histoire qu'ils font. De là, pour une large part, le caractère irritant de La Révolution introuvable : ce que l'on accepte aisément de Tocqueville se faisant, plus d'un demi-siècle après la Révolution, l'historien de l'Ancien Régime, on l'admet beaucoup moins volontiers du spectateur de Mai se constituant dès juillet 1968, à l'égard d'A. Peyrefitte ou de D. Cohn-Bendit, en historien du présent. Une telle réaction, aisément compréhensible,

6 Mémoires, p. 479.

7 Révolution introuvable, p. 33.

$8 \mathrm{Ibid}$. Aron a même cherché à mobiliser pour ainsi dire au sens propre le regard de Tocqueville, puisque, le 29 mai, un de ses premiers articles du Figaro consacré à la crise est en fait un montage de citations de Tocqueville sur février 1848 (repris in Révolution introuvable, p. 161-162). Voir aussi l'article du 10 juin (ibid., p. 167-169). 9 Ibid., p. 29. Cf. aussi la traduction méthodologique du présupposé, p. 115 : «Je n'accorde pas une grande importance, je vous l'ai dit, en période révolutionnaire, aux propos que tiennent les acteurs.»

10 Cité par R. Aron, Etapes de la pensée sociologique, Gallimard, « Tel », p. 250. 
éclaire partiellement la difficile réception de $L a$ Révolution introuvable, sans en être pour autant légitimée : la dimension de sévérité à l'égard des acteurs est ici inhérente au point de vue adopté, qui dès lors devrait être discuté comme tel et à travers les effets d'intelligibilité qu'il procure et non à travers ses éventuels effets pervers sur les susceptibilités des "combattants». C'est en tout cas à ce postulat del'aveuglement des acteurs qu'il convient de rapporter les notions, si mal reçues, de «psychodrame», de «comédie» ou «carnaval»: on peut certes les estimer excessivement désobligeantes et malencontreuses dans leur forme $^{11}$, mais elles participent clairement $\mathrm{du}$ point de vue tocquevillien adopté ${ }^{12}$, puisque, là où les sujets agissants ne sont que les acteurs d'une intrigue tramée sans eux, les idées de «rôles» et de «masques», donc de «psychodrame» et de «carnaval», s'introduisent sans peine ${ }^{13}$.

Si l'on entend lire Mai 1968 avec les yeux de Tocqueville, on présuppose également qu'il ne saurait s'agir là d'une "révolution " au sens d'une brisure dans la continuité historique: contre les illusions des révolutionnaires, Tocqueville avait rétabli la continuité entre l'Ancien Régime et la Révolution, et de même avait vu poindre, dans l'apparente rupture antimonarchique de février 1848, le renforcement bonapartiste de l'autorité de l'Etat; face à Mai, Aron/Tocqueville va récuser qu'il s'agisse d'autre chose que de l'accomplissement d'une logique échappant aux acteurs : de là le thème d'une "révolution introuvable», parfaitement antithétique de l'interprétation de Mai comme «mouvement révolutionnaire», défendue avec conviction par C. Castoriadis, C. Lefort et E. Morin dans La brèche, auxquels on serait alors tenté, du point de vue d'Aron/Tocqueville, d'attribuer dans le psychodrame le rôle d'Ampère ${ }^{14}$. Par là s'explique aussi tout l'effort déployé par Aron pour enlever à la crise son caractère pathétique: loin de constituer la «fin d'une civilisation» ${ }^{15}$ ou la «fin d'un monde» ${ }^{16}$, la séquence, au risque de voir " rétrécir» sa portée, devra être interprétée comme une phase de régulation dans l'autodéveloppement d'une société ce pour quoi aussi sera proposée l'idée de psychodrame, entendue cette fois moins dans sa dimension théâtrale que dans sa portée curative ou thérapeutique ${ }^{17}$. Sans doute cette dernière hypothèse (celle de Mai comme défoulement ou comme " accès de fièvre ») n'est-elle pas, dans les explications que recherche Aron pendant l'été 1968, la plus féconde ni la plus profonde ${ }^{18}$ : elle intervient en tout cas comme l'une des manières possibles de mettre en le postulat de continuité tel qu'il dissout le caractère véritablement «révolutionnaire» de l'événement.

Le point de vue selon lequel les acteurs de Mai ont joué un épisode dont la logique leur

11 Cf. Aron lui-même, Révolution introuvable, p. 33 : «Je ne maintiens pas sans nuances cette expression» (de psychodrame, utilisée à Radio-Luxembourg, le 1er juin).

12 Encore participeraient-elles tout autant, pour des raisons analogues, de l'adoption d'un point de vue marxiste (cf. la remarque de Marx, au début du 18 Brumaire de Louis-Napoléon Bonaparte, selon laquelle " tous les grands événements historiques se répètent pour ainsi dire deux fois... la première fois comme tragédie, la seconde fois comme farce »).

13 Cf. aussi, à cet égard, le vocabulaire cinématographique utilisé in Révolution introuvable, p. 141 sqq. : film, séquence, scénario, rôle, etc.

14 Cf. par exemple C. Castoriadis (J.-M. Coudray), La brèche, p. 92 : le mouvement opère " l'affirmation révolutionnaire la plus radicale ", procédant d'une volonté de "rupture radicale avec le monde capitaliste bureaucratique » et de « reconstitution révolutionnaire de la société ». Sur l' « irréductible opposition » entre $L a$ brèche et La Révolution introuvable, cf. Révolution introuvable, p. 12 sqq.

15 Révolution introuvable, p. 19 sqq. ; cf. aussi p. 44 sqq.

16 Ibid., p. 31 sqq.

17 Ibid., p. 47.

18 Dans les Mémoires (p. 479), elle n'est évoquée que brièvement, comme une perspective parmi beaucoup d'autres : «Cette soudaine diversion à l'ennui quotidien... » 
échappait impose enfin un dernier postulat, quant à la nature même ou au contenu même de cette logique ${ }^{19}$.

La Révolution introuvable esquisse à cet égard deux niveaux d'hypothèses interprétatives, selon qu'Aron se place au plan de l'histoire partielle ou prochaine (celle de la Ve République) ou au plan de l'histoire globale ou lointaine (celle de la modernité) : 1 / Au niveau de l'histoire prochaine, la logique identifiée est, on l'a entrevu ci-dessus, celle d'un renforcement ambigu du pouvoir gaulliste, comme «régime de droite renforcé» (par sa majorité), mais " affaibli par la résistance d'une partie du pays»: à l'évidence prophétique moins d'un an avant l'échec du référendum de 1969, ce premier diagnostic continuiste ne confère cependant à l'événement qu'une signification proprement « politicienne » qui ne saurait à elle seule ni épuiser la diversité des aspects de la crise (notamment ses aspects culturels), ni désigner la logique ultime d'un devenir historique irréductible à ce qu'en exprime la succession des majorités politiques.

2/Il est donc heureux que l'interprétation se soit complétée au niveau de l'histoire globale comprise comme histoire de la modernité. Anticipant sur ce qu'allait être l'avenir des «soixante-huitards», La Révolution introuvable suggère en effet que, dans les cas les plus favorables, l'issue sera trouvée, non pas dans l'adhésion à un «ordre de remplacement» (projet politique, idéal collectif), mais «par la démission, l'indifférence, la fuite vers la résidence secondaire ${ }^{20}$. A l'horizon de la «révolution (introuvable) de Mai» se profileraient donc la désertion du politique, le repli sur la sphère privée, l'essor d'un individualisme hédoniste neutralisant les grands débats publics et chômant les valeurs du collectif. Là encore, le caractère prophétique de l'interprétation apparaît comme singulièrement saisissant et, dans une certaine mesure, il n'est pas excessif de souligner qu'Aron esquisse ainsi, dès l'été 1968, l'une des pistes reconnues aujourd'hui, notamment à travers le livre de G. Lipovetsky, parmi les plus fécondes dans la recherche du sens de Mai. Et il n'est pas besoin d'insister longuement sur la manière dont, ce faisant, Aron assume jusqu'au bout le choix d'un point de vue tocquevillien, puisqu'aussi bien, comme il le montre lui-même dans sa présentation de Tocqueville ${ }^{21}$, l'installation $\mathrm{du}$ travail de l'individualisme au coeur de la modernité démocratique constitue sans doute l'apport le plus spécifique et le plus enrichissant de Tocqueville à l'archéologie de notre présent. J'ajoute que cette inscription de Mai 68 dans la logique de l'individualisme démocratique permet à l'analyse de prendre ici en compte, parmi les composantes multiples de la période, le climat intellectuel dominant: «L'intelligentsia des années soixante, écrit Aron, avait pour dieu, non plus le Sartre de l'après-guerre, mais un mélange de Lévi-Strauss, Foucault, Althusser et Lacan» ${ }^{22}$, dont les pensées et les pratiques (théoriques) convergeaient (par-delà tout ce qui les distinguait) vers la production de deux types d'effets : elles contribuaient à engendrer la conviction qu' «Il n'y a pas de faits» (mais seulement des interprétations), et donc à dissoudre la conviction raisonnable selon laquelle " toute société est soumise à des contraintes de fait, la nécessité de la production, de l'organisation d'une hiérarchie technique, la nécessité d'une technobureaucratie et ainsi de suite ${ }^{23}$ : d'une telle dissolution des faits et de leurs contraintes devait résulter progressivement (outre la fascination pour l'utopie) la conviction que tout se vaut et que, par exemple sur le plan

19 Je ne reviens pas ici sur la question du statut aronien (ou tocquevillien) de cette logique. Pour des raisons qu'a thématisées dès 1938 l'Introduction à la philosophie de l'histoire, il est clair que la logique décelée est davantage un point de vue qu'une thèse sur la réalité en soi du devenir historique. Sur les conséquences de cette option théorique, cf. L. Ferry et A. Renaut, La Pensée 68, Gallimard, 1985, p. 93 sqq. (« Pour le pluralisme interprétatif»). 20 Révolution introuvable, p. 137.

21 Etapes de la pensée sociologique, p. 224 sqq.

22 Révolution introuvable, p. 136.

23 Ibid., p. 123. 
éthico-juridique, Il n'est pas de normes qui se doivent imposer au jeu du désir ; d'autre part, à travers cette dissolution des normes, c'est un néo-nihilisme qui se profile, minant d'autant plus aisément l'ordre déjà fragilisé d'une société fondée sur des normes souvent désuètes, en tout cas «sans âme»: dans ce "refus d'un ordre sans vision d'un ordre de remplacement », on tient d'ailleurs sans doute aussi, suggère Aron, $l^{\prime}$ « une des causes de la décomposition de Mai $»^{24}$.

Je ne prétends évidemment pas que $L a$ Révolution introuvable accomplisse pleinement cette mise en relation, qui requiert d'être subtile et nuancée, de la crise de Mai, comme destruction des normes ("Il est interdit d'interdire »), avec le travail critique accompli par la philosophie française des «sixties » à l'égard d'une normativité dénoncée comme répressive en tant que telle. Pas plus qu'il y a un instant je n'estimais achevée par R. Aron la lecture individualiste de Mai 68. Il n'en demeure pas moins, je le répète, que des pistes se trouvent là indiquées, qui interdisent de considérer décidément $L a$ Révolution introuvable comme le simple mouvement d'humeur d'un «mandarin».

Ainsi se laissent mettre en place, à partir de La Révolution introuvable, les principes d'une interprétation de Mai 68 qui inscrirait la crise dans la logique de la modernité démocratique et individualiste. A jouer le rôle de Tocqueville plutôt que celui, plus facile à assumer, d'Ampère, R. Aron conduit, de fait, les futurs interprètes de Mai à prendre en compte ce singulier paradoxe: à travers un mouvement qui, en un sens, s'est voulu antidémocratique (au sens où il a mis en cause au moins la forme la plus généralement moderne de l'idée démocratique, à savoir l'idée de démocratie représentative), s'est déployée la logique de la modernité démocratique comme cette égalisation des conditions dont l'individualisme est indissolublement la cause et l'effet. La critique peu responsable de l'idée d'élection ( "élection = trahison $)^{25}$ s'est en effet, en Mai 68, aventurée sur des terrains où les bases de la « légitimité démocratique » risquaient de se voir sapées et où pouvait se produire ce que les Mémoires nomment une "rupture de la légalité républicaine» ${ }^{26}$. Face au risque d'une telle «rupture», Aron n'hésite pas en 1968 à exprimer un rejet quasiment physique, dont la radicalité est sans doute elle aussi l'une des explications du ton adopté par La Révolution introuvable et de son absence de toute concession à l'égard du mouvement: «Edgar Morin sympathise avec la Commune estudiantine qui m'inspire une répulsion immédiate: on ne discute pas des goûts et des couleurs ${ }^{27}$. Quinze ans après, le ton s'est apaisé, mais ce qui était à son principe demeure : «Une rupture de la légalité républicaine, dix ans après le coup d'Etat légal de 1958, m'apparaissait déplorable. Je n'étais certes pas un gaulliste inconditionnel, mais la victoire de Cohn-Bendit sur le général m'aurait blessé en profondeur».

Là sans doute, dans l'adhésion infrangible ou, au contraire, dans le refus plus ou moins assumé de la légitimité démocratique moderne (synthèse démocratico-libérale, selon l'expression de Démocratie et totalitarisme, ou, si l'on veut, synthèse démocratico-républicaine, en tout cas régime démocratique au sens de la démocratie représentative des régimes constitutionnelspluralistes), réside le fondement ultime des positions parfois extrêmes prises vis-à-vis du mouvement de Mai que ce soit sur le mode de la " répulsion immédiate " (Aron) ou sur le mode de l'enthousiasme débordant ${ }^{28}$. Si telle devait apparaître la vérité ultime de ces prises de position (en tout cas quand elles se sont réfléchies et ont entrepris de se légitimer politiquement),

24 Ibid., p. 137.

25 Sur ce slogan de Mai, cf. Plaidoyer pour l'Europe décadente, p. 506.

26 Mémoires, p. 493.

27 Révolution introuvable, p. 14.

28 Je pense bien sûr à $C$. Castoriadis, dont l'enthousiasme pour Mai ne peut guère être séparé de sa fascination pour le modèle de la démocratie directe : cf. encore, tout récemment, Sur la démocratie grecque, Le Débat, janvier-mars 1986. 
resterait alors à évaluer ce qui, de part et d'autre de cette ligne de partage, en résulte pour la validité des deux types d'attitude.

Je me bornerai à deux remarques délibérément succinctes : 1 . Nul n'est à vrai dire absolument forcé d'être convaincu que, face aux imperfections manifestes de la démocratie représentative des Modernes, les principes de la démocratie grecque telle que Castoriadis y voit le modèle d'une démocratie directe soient compatibles à la fois avec les conditions de notre vision du monde et avec les exigences minimales qui définissent pour nous le respect de la personne humaine.

2. Même là où la «répulsion immédiate» à l'encontre d'une éventuelle rupture de la «légalité républicaine» a pu conduire à ne pas toujours mesurer à leur juste prix certains aspects d'un mouvement au demeurant très hétérogène, il reste que la prise en compte de la problématique de la démocratie moderne (comme problématique de l'égalité) a pu ne pas interdire, au terme de l'analyse d'un moment paradoxalement antidémocratique de la logique de la modernité démocratique, d'y voir une étape d'un progrès vers un ordre différant «en mieux de l'ordre ancien». 


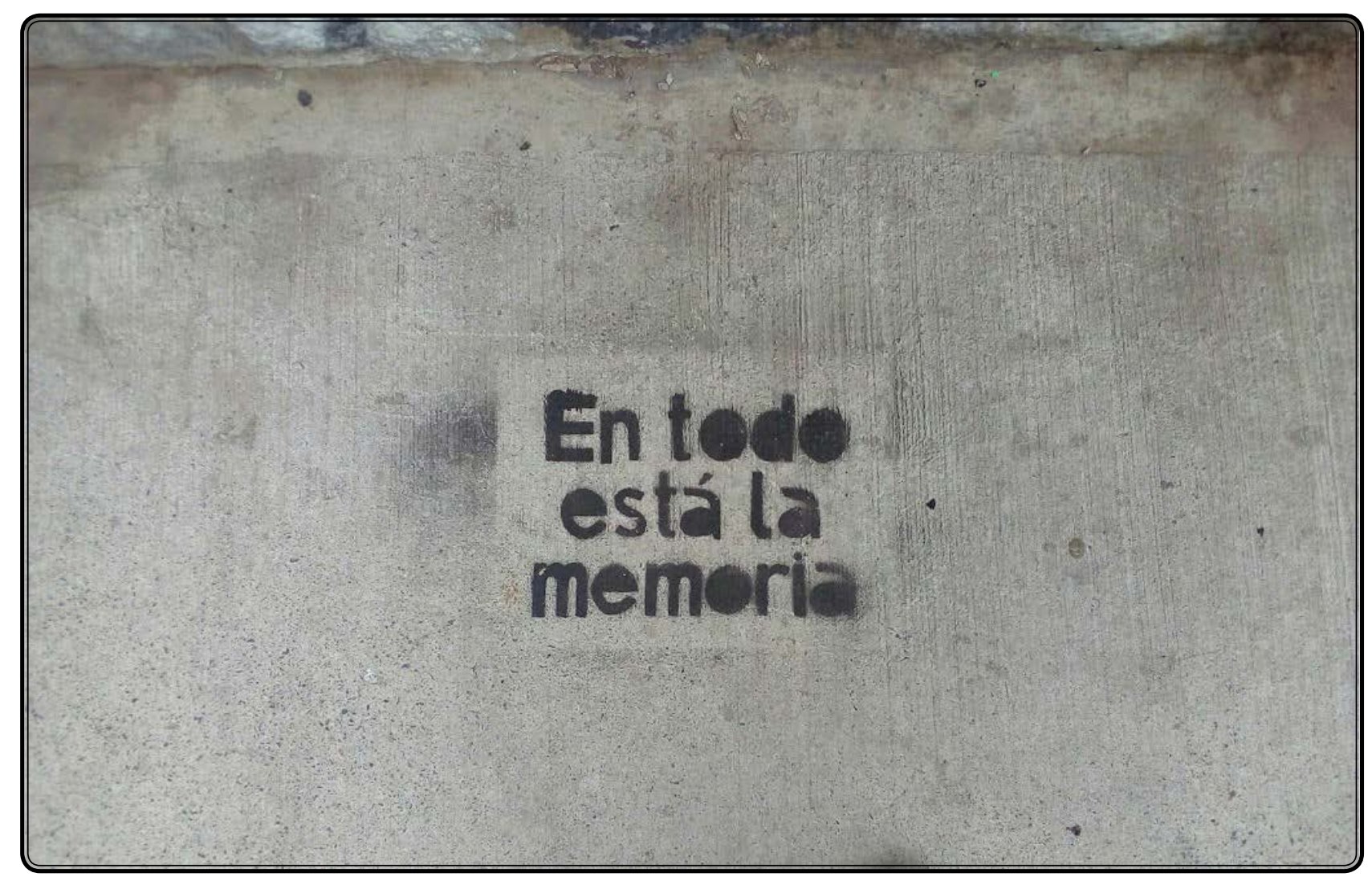

Sin lugar para el olvido. 